\title{
Follow-up analysis of a randomized phase III immunotherapeutic clinical trial on melanoma
}

\author{
ROBERT SURIANO $^{1}$, SHILPI RAJORIA ${ }^{1}$, ANDREA L. GEORGE ${ }^{1}$, JAN GELIEBTER ${ }^{1}$, \\ RAJ K. TIWARI ${ }^{1}$ and MARC WALLACK ${ }^{1,2}$ \\ ${ }^{1}$ Department of Microbiology and Immunology, New York Medical College, Valhalla, NY 10595; \\ ${ }^{2}$ Department of Surgery, Metropolitan Hospital Center GNS, New York, NY 10029, USA
}

Received November 27, 2012; Accepted February 2, 2013

DOI: $10.3892 / \mathrm{mco} .2013 .97$

\begin{abstract}
Development of a melanoma-specific vaccine is of clinical necessity. Therefore, a phase III, randomized, double-blind trial was performed (June 1988-June 1991) to assess the clinical effectiveness of our vaccinia melanoma oncolysate (VMO) vaccine in stage III melanoma patients. Patient data were collected from 11 institutions, as well as from the Social Security Death Index and were analyzed from April through August 2008 for disease-free interval (DFI) and overall survival (OS). The median OS for patients who were administered the VMO vaccine was 7.71 years, compared to 7.95 years for patients administered the vaccinia virus vaccine $(\mathrm{V})(\mathrm{p}=0.70)$. The median DFI for the VMO group was six years, while the median DFI for the $\mathrm{V}$ group has not yet been reached. This analysis demonstrated a statistically significant difference in OS in females in both groups (VMO, 79\%; V, 92\%), as compared to males (VMO, 57\%; $\mathrm{V}, 68 \%)(\mathrm{p}=0.0473)$. This follow-up analysis demonstrated that females had a survival advantage over males, thus warranting further investigation. This significant observation may facilitate the recruitment of patients for future clinical trials, as well as determine which patients are more likely to benefit from receiving the VMO vaccine.
\end{abstract}

\section{Introduction}

Melanoma arises from the transformation of the pigmentproducing melanocytes of the skin and is often initiated by deregulation of the MAPK pathway, which is known to modulate melanoma cell survival $(1,2)$. According to the National Cancer Institute (NCI), melanoma remains the most fatal type of skin cancer and is the fifth most commonly occurring cancer in men and the seventh in women. In 2011, it was estimated that

Correspondence to: Dr Marc Wallack, Department of Surgery, Metropolitan Hospital Center, 1901 1st Avenue, New York, NY 10029, USA

E-mail: marc.wallack@nychhc.org

Key words: melanoma, vaccine, vaccinia virus, immunotherapy over 70,000 individuals would be diagnosed with melanoma and approximately 9,000 individuals would succumb to the disease in the USA (3). Surgery remains the standard treatment for localized melanoma skin lesions, whereas the standard of care for metastatic melanoma remains a single chemotherapeutic agent, known as dacarbazine, which has a response rate of $10-15 \%$ in patients and is not associated with long-lasting responses, since the median survival rate is approximately nine months $(4,5)$. This lack of effective chemotherapeutic compounds has led researchers to focus on the development of immunotherapeutic methods to treat melanoma.

Immunotherapeutic melanoma-treatment strategies have been investigated over the past 30 years and resulted in various clinical trials (6). Immunotherapy has focused on four major areas: immuno-activating cytokines, immunomodulating antibodies, adoptive T-cell therapy and vaccines. The immuno-activating cytokine, interleukin-2 (IL-2), is one of three FDA-approved compounds used to treat melanoma; it is, however, associated with high toxicity (7). The FDA has recently approved an immunotherapeutic compound that targets melanoma, known as ipilimumab (anti-CTLA-4 antibody) (8), which has shown promising results in clinical trials. In addition, although it is not an immunotherapeutic compound, it should be noted that the FDA has approved a small molecule inhibitor, known as vemurafenib (BRAF inhibitor) (9), which has also shown promising results in clinical trials. Although there are ongoing clinical trials aimed at assessing the effectiveness of various immunotherapeutic compounds, further research on melanoma vaccines is required, since melanoma is one of the most immunogenic types of cancer and several clinically relevant melanoma-associated antigens (MAAs) have already been identified (10-13).

The possibilities for developing antigen-based vaccines against melanoma are numerous, as multiple MAAs have been identified since the 1970s, cloned $(14,15)$ and well-characterized, in vivo and in vitro, with respect to their antigenicity. Despite their characterization, not all antigens elicit an optimal immune response, which may be attributed to the fact that the selected antigens were not HLA-typed to match the vaccinated individuals or that an adjuvant is required to help initiate an antigen-specific immune response. Examples of antigens used in vaccines against melanoma are MAAs such as gp100, tyrosinase, MAGE-3 and MART-1 (16-18); however, phase I and II 
studies have not shown promising results when administered as a single antigen/protein-containing vaccine $(19,20)$. Other clinical trials have focused on the generation of vaccines that incorporate one or more antigens, combined with the administration of an immuno-activating cytokine, such as IL-2 or granulocyte-macrophage colony-stimulating factor (GM-CSF) $(19,21,22)$. One such clinical trial recently showed a high response rate and progression-free survival in patients with advanced melanoma who were vaccinated with gp100 and IL-2 (23). Melanoma vaccines using a multivalent antigen approach appear promising, although further studies should be performed to determine the conditions that are likely to foster a productive immune response, such as selecting an adjuvant with optimal immuno-stimulatory properties.

Previously, we developed a melanoma multivalent vaccine using live vaccinia virus-augmented melanoma cell lysates, in order to generate the vaccinia melanoma oncolysate (VMO), or first generation melanoma vaccine. The VMO is composed of four established allogeneic melanoma cell lines, providing a variety of MAAs that help generate a robust immune response, using vaccinia virus as an adjuvant $(24,25)$. Vaccinia virus was incorporated as an adjuvant in VMO since i) it modifies and re-expresses host membrane-associated melanoma antigens, thus making these antigens more recognizable to the immune system of the host (26) and ii) it non-specifically induces tumor-specific cytotoxic T lymphocytes (CTLs), thus initiating the host immune response (27).

The efficacy of VMO in the treatment of melanoma was studied in several preliminary trials $(24,28)$, followed by the Southeastern Cancer Study Group-sponsored phase I and II trials $(29,30)$. Given the favorable results of these trials, we proceeded with the first randomized, prospective, doubleblind, multi-institutional, pharma-produced, FDA-approved, NCI-funded melanoma vaccine phase III trial (1988-1991), in order to evaluate the effects of VMO on disease-free interval (DFI) and overall survival (OS) in patients with stage II melanoma (based on the International Union Against Cancer criteria). Although the results of the first interim analysis (performed in May 1994) showed no statistical difference in OS between patients receiving VMO and those receiving the control vaccinia virus vaccine (V), there was a $10 \%$ increased survival advantage in favor of VMO. Moreover, although the number of patients that were enrolled in this trial $(n=250)$ was not sufficient to generate statistically significant results in specific subset analyses, the final analysis demonstrated increased OS in VMO-treated patients, specifically in i) VMO-treated males, aged 44-57 years, with one to five positive nodes and ii) VMO-treated males and females who had undergone prophylactic lymph node dissection which demonstrated positive nodes (clinical stage I and pathological stage II disease) (31). A second interim analysis (performed in May 1995) demonstrated no difference in survival between VMOand V-treated groups; however, further analysis revealed i) a subset of males aged 44-57 years, with one to five positive nodes, treated with VMO, that had a statistically significant OS compared to those treated with the placebo $\mathrm{V}(\mathrm{p}=0.037)$ and ii) patients with clinical stage I disease, treated with VMO, that had a statistically significant OS compared to those treated with the placebo $\mathrm{V}(\mathrm{p}=0.05)(32)$. The third analysis of this phase III trial (performed in May 1996) showed no significant difference in either DFI or OS in patients treated with VMO, compared to those treated with V; however, the same subset of males, aged 44-57 years, with one to five positive nodes, treated with VMO, showed a statistically significant improvement in survival at two-, three- and five-year intervals ( $\mathrm{p}=0.046)$.

Based on the results and trends observed in our clinical trial, we report on a 10-year follow-up analysis on the patients originally involved in the phase III trial. We have now compiled follow-up data on the surviving patients originally enrolled in the phase III trial and present data that reflect upon the validity and efficacy of the trial design originally envisioned.

\section{Materials and methods}

Patients. As previously described (31-33), 250 patients were accrued from 11 participating institutions, from June 1988 to January 1991. These institutions are listed as follows: MD Anderson Cancer Center, Houston, TX; University of Alabama, Birmingham, AL; Emory University, Atlanta, GA; University of Pennsylvania, Philadelphia, PA; Wayne State University, Detroit, MI; University of Florida, Gainesville, FL; University of Chicago, Chicago, IL; University of Colorado, Denver, CO; Duke University, Durham, NC; Brown University, Providence, RI; and Mount Sinai Medical Center, Miami Beach, FL, USA. Patients were selected according to strict eligibility criteria, which included histologically-positive nodes, age range 15-70 years, Karnofsky performance status $<70 \%$ and no concomitant malignancy, apart from basal and squamous cell carcinoma of the skin. Originally, patients with any number of positive nodes were eligible for the trial during the first year (June 1988-June 1989). Subsequently, only patients with one to five positive nodes were accepted, since the protocol advisory panel (including Dr Charles Balch, statistician; Dr Al Bartolucci, medical advisor; and Dr Marc Wallack, principal investigator) considered more than five positive nodes to represent excessive tumor burden. Further criteria for ineligibility included patients with: lentigo maligna melanoma; satellite lesions at a distance $>2 \mathrm{~cm}$ from the primary lesion; previous chemotherapy, radiotherapy, or immunotherapy; matted nodes (except during the first year of the trial); more than one primary lesion; and pregnancy. Patients were enrolled in the study eight weeks after surgery.

Informed consent was obtained from all patients enrolled in our study and the procedures followed were in accordance with the ethical standards of the responsible committee on human experimentation and with the Helsinki Declaration of 1975, as revised in 1983 .

VMO, V and attenuated smallpox preparation. As previously described (31-33), the VMO vaccine for the treatment arm, the $\mathrm{V}$ vaccine for the control arm and the smallpox vaccine for both arms (used as an immune booster), were prepared by the Mérieux Institute, Lyon, France. The preparation of the VMO has been extensively described in our previous publication (31). Essentially, four allogeneic cell lines (Mel-2, Mel-3, Mel-4 and Mel-B) were established from four patients treated in our clinic. These cell lines were assessed using standard assays to confirm the absence of bacteria, fungi, mycoplasma and all human viruses (including human immunodeficiency virus). 
The $\mathrm{V}$ vaccine was prepared by infecting the MRC5 human diploid cell line with a seed vaccinia virus that was developed in this laboratory, using smallpox vaccine 'Dryvax' (Wyeth Laboratories, Inc., Marietta, PA, USA), containing the New York City Board of Health strain of vaccinia virus. Melanoma cells were infected with the vaccinia virus at a ratio of 1 cell to $10 \mathrm{TCID}_{50}(50 \%$ tissue culture infectious dose) of vaccinia virus, then incubated overnight at $37^{\circ} \mathrm{C}$. Virus-infected cells were lysed by sonication and a nucleus-free cell lysate was obtained by centrifugation to create the final product, VMO.

Study enrollment. Surgical review of the wide excision and regional lymphadenectomy was performed for each patient prior to registration. Pathological review of the biopsy specimens was performed by the study pathologist to establish the diagnosis of stage III melanoma (based on 1988 AJCC criteria). All the patients had complete physicals and laboratory workup, including complete blood count, liver function tests and urinalysis, as well as baseline chest X-ray. Each patient signed an informed consent form, that was approved by the respective institution. Patients were then randomized between the VMO and V treatment groups by the Statistical Center at the University of Alabama.

$V M O, V$ and attenuated smallpox vaccine administration. The administration of the respective vaccines has been described in detail in previous publications (31-33). Briefly, each patient was given a smallpox booster injection subcutaneously $\geq 48 \mathrm{~h}$ prior to the initiation of treatment. The VMO or V was injected intradermally into the sites in proximity to the regional lymph node groups, excluding the sites of node dissections. One dose of $\mathrm{VMO}$ or $\mathrm{V}$ was divided into four to six aliquots, with two to four aliquots injected above the waist in the infraclavicular region bilaterally and two aliquots injected below the waist in the infrainguinal region bilaterally. These injections were given once a week for 13 weeks, then once every two weeks for one year or until recurrence. These patients were closely monitored throughout the trial for signs and symptoms of toxicity, as well as recurrence.

Statistical analysis. For the follow-up analysis, the Social Security Death Index was accessed to obtain additional information on the patients. Additionally, each institution involved in the original trial was contacted regarding the status of these patients, as well as any additional therapies these patients may have received after VMO. Statistical analysis was performed by the Statistical Center at the Department of Biostatistics, University of Alabama, using log-rank statistics. Patient data were analyzed using 2002 AJCC staging criteria.

\section{Results}

Patient characteristics and survival analysis. Records were available for 109 out of the 111 patients evaluated. Patient characteristics of the $\mathrm{VMO}$ group and the $\mathrm{V}$ group are presented in Table I. In April 2008, 78 patients were still alive in both VMO and V groups, with 35 surviving patients $(45 \%)$ in the VMO group and $43(55 \%)$ in the V group. Thirty-one patients (19 patients in the VMO group and 12 in the V group) had
Table I. Patient physical data comparing VMO and V.

\begin{tabular}{|c|c|c|}
\hline & VMO & $\mathrm{V}$ \\
\hline Number of patients & 54 & 55 \\
\hline $\begin{array}{l}\text { Number of patients alive } \\
\text { (April 2008) }\end{array}$ & 35 & 43 \\
\hline Gender & 35 & 19 \\
\hline Male & 35 & 31 \\
\hline Female & 19 & 24 \\
\hline \multicolumn{3}{|l|}{ Primary tumor site } \\
\hline Head/neck & 4 & 3 \\
\hline Upper extremity & 13 & 11 \\
\hline Trunk & 21 & 28 \\
\hline Lower extremity & 8 & 8 \\
\hline Unknown & 8 & 3 \\
\hline \multicolumn{3}{|l|}{ Type of melanoma } \\
\hline Nodular & 14 & 13 \\
\hline Radial & 23 & 25 \\
\hline Other (incl. lentigo maligna) & 7 & 4 \\
\hline Unknown & 10 & 13 \\
\hline \multicolumn{3}{|l|}{ Ulceration } \\
\hline Absent & 34 & 33 \\
\hline Present & 12 & 18 \\
\hline Unknown & 8 & 4 \\
\hline \multicolumn{3}{|l|}{ Location of lymph nodes } \\
\hline Axillary & 37 & 38 \\
\hline Cervical & 7 & 3 \\
\hline Inguinal & 10 & 14 \\
\hline \multicolumn{3}{|l|}{ Nodal status } \\
\hline Macroscopic & 40 & 38 \\
\hline Microscopic & 14 & 17 \\
\hline \multicolumn{3}{|l|}{$\begin{array}{l}\text { Clinical stage } \\
\text { (based on } 2002 \text { AJCC criteria) }\end{array}$} \\
\hline IIIA & 10 & 7 \\
\hline IIIB & 22 & 33 \\
\hline IIIC & 22 & 15 \\
\hline \multicolumn{3}{|l|}{ Disease status } \\
\hline NED & 41 & 44 \\
\hline REC & 13 & 11 \\
\hline
\end{tabular}

VMO, vaccinia melanoma oncolysate vaccine; $\mathrm{V}$, vaccinia virus vaccine; NED, no evaluable disease; REC, recurrence.

succumbed in the time period between this analysis and the third analysis (performed in May 1996).

The VMO and V groups were further subdivided into alive and deceased patient subsets $\left(\mathrm{VMO}_{\text {alive }}, \mathrm{VMO}_{\text {deceased }}, \mathrm{V}_{\text {alive }}\right.$ and $\left.\mathrm{V}_{\text {deceased }}\right)$ and the demographics of these respective subsets are presented in Table II. There was no significant difference in distribution of age $(\mathrm{p}=0.3286)$, number of nodes $(\mathrm{p}=0.6855)$, time $(\mathrm{p}=0.5525)$, or size of primary lesion $(\mathrm{p}=0.4008)$ among $\mathrm{VMO}_{\text {alive }}, \mathrm{VMO}_{\text {deceased }}, \mathrm{V}_{\text {alive }}$ and $\mathrm{V}_{\text {deceased }}$ groups. Subsequent subset analysis revealed a statistically significant survival advantage in females, regardless of whether they were administered VMO or V $(\mathrm{p}=0.047)$. However, there was no 
Table II. Patient physical data for VMO and V alive, compared to VMO and V deceased patient subsets.

\begin{tabular}{|c|c|c|c|c|}
\hline & $\mathrm{VMO}_{\text {alive }}$ & $\mathrm{VMO}_{\text {deceased }}$ & $\mathrm{V}_{\text {alive }}$ & $\mathrm{V}_{\text {deceased }}$ \\
\hline Number of patients & 35 & 19 & 43 & 12 \\
\hline \multicolumn{5}{|l|}{ Gender } \\
\hline Male & 20 & 15 & 21 & 10 \\
\hline Female & 15 & 4 & 22 & 2 \\
\hline \multicolumn{5}{|l|}{ Primary tumor site } \\
\hline Head/neck & 2 & 2 & 2 & 1 \\
\hline Upper extremity & 6 & 2 & 6 & 2 \\
\hline Trunk & 13 & 8 & 13 & 7 \\
\hline Lower extremity & 5 & 3 & 5 & 0 \\
\hline Unknown & 9 & 4 & 11 & 2 \\
\hline \multicolumn{5}{|l|}{ Ulceration } \\
\hline Absent & 23 & 11 & 23 & 10 \\
\hline Present & 7 & 5 & 16 & 2 \\
\hline Unknown & 5 & 3 & 4 & 0 \\
\hline \multicolumn{5}{|c|}{ Location of lymph nodes } \\
\hline Axillary & 23 & 14 & 30 & 8 \\
\hline Cervical & 5 & 2 & 2 & 1 \\
\hline Inguinal & 7 & 3 & 11 & 3 \\
\hline \multicolumn{5}{|l|}{ Nodal status } \\
\hline Macroscopic & 28 & 12 & 33 & 5 \\
\hline Microscopic & 7 & 7 & 10 & 7 \\
\hline \multicolumn{5}{|c|}{$\begin{array}{l}\text { Clinical stage } \\
\text { (based on } 2002 \text { AJCC criteria) }\end{array}$} \\
\hline IIIA & 6 & 4 & 3 & 4 \\
\hline IIIB & 14 & 8 & 26 & 7 \\
\hline IIIC & 15 & 7 & 14 & 1 \\
\hline \multicolumn{5}{|l|}{ Disease status } \\
\hline NED & 31 & 10 & 37 & 7 \\
\hline REC & 4 & 9 & 6 & 5 \\
\hline \multicolumn{5}{|l|}{ Age } \\
\hline Males 44-57 years & 7 & 5 & 2 & 3 \\
\hline Other & 28 & 14 & 41 & 9 \\
\hline
\end{tabular}

VMO, vaccinia melanoma oncolysate vaccine; $\mathrm{V}$, vaccinia virus vaccine; NED, no evaluable disease; REC, recurrence.

statistically significant association between type of melanoma $(\mathrm{p}=0.9311)$, ulceration $(\mathrm{p}=0.3798)$, node location $(\mathrm{p}=0.8303)$, disease status $(\mathrm{p}=0.0521)$, stage $(\mathrm{p}=0.1198)$, location of primary lesion ( $\mathrm{p}=0.9295$ ) or age (specifically, males aged $44-57$ years) with survivability among the $\mathrm{VMO}_{\text {alive }}, \mathrm{VMO}_{\text {deceased }}, \mathrm{V}_{\text {alive }}$ and $\mathrm{V}_{\text {deceased }}$ groups.

Additional subset analysis, evaluating melanoma stage and disease spread, demonstrated a statistically significant association between melanoma stage and nodal status (i.e., microscopic vs. macroscopic disease) $(p=0.0001)$, with a higher incidence of microscopic disease in stage IIIA patients and a higher incidence of macroscopic disease in stage IIIC patients. However, there was no statistically significant association between melanoma stage and node location $(p=0.8641)$, thus demonstrating that the location of the melanoma-positive lymph node basins had no impact on the OS of melanoma.
As shown in Fig. 1, there was no statistically significant difference in DFI between patients receiving VMO and those receiving $\mathrm{V}(\mathrm{p}=0.76)$. The median DFI for the VMO group was six years, while the median DFI for the $\mathrm{V}$ group has not yet been reached.

The OS of patients treated with VMO or $\mathrm{V}$ is shown in Fig. 2. In patients administered VMO, the median OS was 7.71 years, compared to 7.95 years in those administered $\mathrm{V}$ $(\mathrm{p}=0.70)$.

\section{Discussion}

Identification of new immunotherapeutic strategies to treat melanoma remains an area of intense research. In addition to the FDA-approved immuno-activating IL-2 (7) and the immuno-modulatory agent ipilimumab (9) as treatments for melanoma patients, research on developing melanoma 


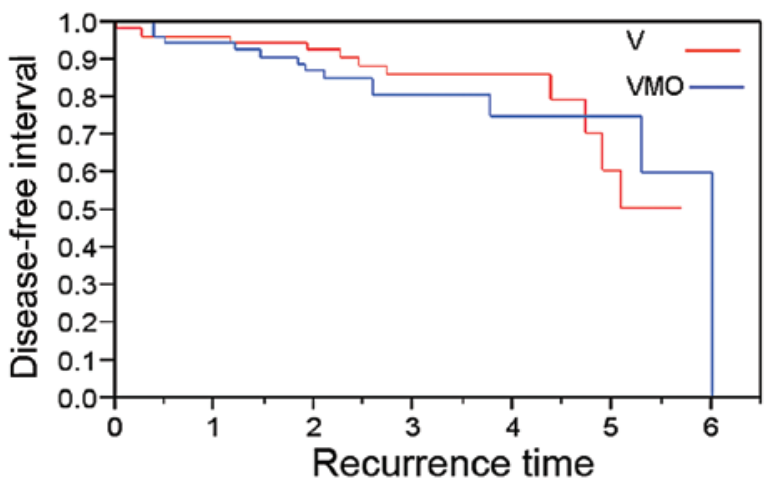

Figure 1. Kaplan-Meier survival plot depicting disease-free interval (DFI) in years. There was no statistically significant difference in DFI between patients receiving vaccinia melanoma oncolysate (VMO) vaccine and those receiving vaccinia virus $(\mathrm{V})$ vaccine $(\mathrm{P}=0.76)$. The median $\mathrm{DFI}$ for the VMO group was six years, while the median DFI for the $\mathrm{V}$ group has not yet been reached.

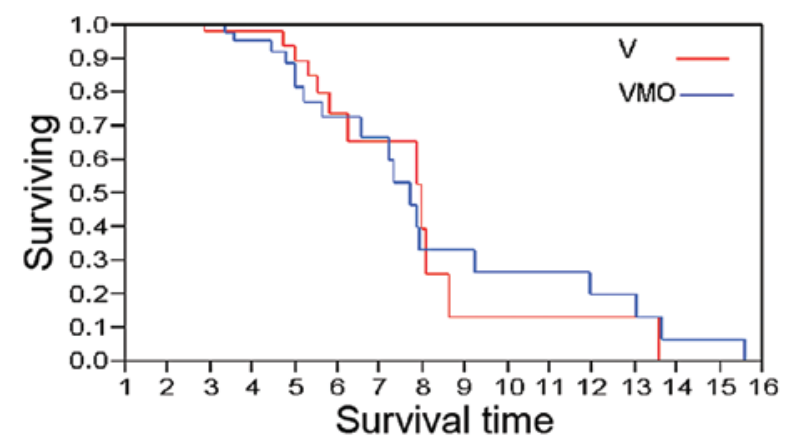

Figure 2. Kaplan-Meier survival plot depicting patient overall survival (OS) in years. There was no statistically significant difference in OS between patients receiving vaccinia melanoma oncolysate (VMO) and those receiving vaccinia virus $(\mathrm{V})(\mathrm{P}=0.6957)$. In patients that received $\mathrm{VMO}$, the median OS was 7.71 years, compared to 7.95 years in those that received $\mathrm{V}(\mathrm{P}=0.70)$.

vaccines remains a field of interest, since melanoma is the only type of cancer which is highly immunogenic, by virtue of its expression of a plethora of MAAs (16-18). Previous studies on identification of melanoma vaccines have focused on either the use of a single MAA, such as gp100 or MART-1, or multiple MAAs, although the optimal conditions required to initiate and sustain an anti-melanoma immune response were not met. Our laboratory has created and characterized a multivalent MAA vaccine using four primary melanoma cell lines and vaccinia virus as a potent adjuvant $(24,25)$, which was evaluated in an FDA-approved, NCI-funded clinical trial.

To the best of our knowledge, our first generation melanoma vaccine (VMO) phase III trial was the first FDA-approved and NCI-funded, randomized, prospective, multi-institutional and double-blind cancer vaccine trial to employ a polyvalent vaccinia virus-augmented melanoma cell lysate against stage III melanoma. This trial was the first cancer vaccine trial designed to assess the efficacy of VMO, without any bias in the selection of patients, distribution of VMO and V and collection or analysis of patient data. Thus, this series is unique, not only in terms of the treatment provided, but also in its design, which was a novel strategy to improve the only standard of care that was previously available, which was surgery alone.
This follow-up analysis demonstrated no differences in DFI or OS in patients who received VMO compared to those who received $\mathrm{V}$, although it should be noted that a significant number of patients from the original trial (78) were still alive at the time of this follow-up analysis. The fact that such a large number of VMO patients (35), as well as V patients, survived, demonstrates the possible benefits of either method of immune stimulation and, more importantly, highlights the longevity of the immune response that was initially generated (as detected by delayed-type hypersensitivity reactions) in both the VMO and $\mathrm{V}$ treatment arms. Furthermore, it should be stated that, at the time of the vetting of this phase III trial, through the FDA and NCI, there was intense discussion on the use of $\mathrm{V}$ as a treatment arm, since standard of care dictated surgery alone as the second arm. More importantly, $\mathrm{V}$ by itself has been shown to produce 'therapeutic efficacy' in melanoma patients (34). In fact, there should not have been any other placebo used in this trial except a true no-treatment arm, as it is our opinion that such a placebo for this trial would have helped elucidate the efficacy of the VMO. Thus, it is plausible that the VMO arm may have demonstrated statistically significant results if a 'true' no-treatment arm was included (35).

Of note, in this follow-up analysis, the phase III trial demonstrated a statistically significant increase in OS in VMO- and V-treated females compared to males in both arms, even though in the first three analyses, males had exhibited an increased OS, particularly the VMO-treated, aged 44-57 years subset. In general, female melanoma patients exhibit significantly increased survival rates compared to males, which is usually attributed to earlier detection among women (36) and it can also be attributed to the fact that this trial did not include a sufficient number of patients, in order to perform significant survival analyses in subsets with prognostic significance. Therefore, we strongly recommend that future ASI trials enroll a sufficient number of patients in each of the melanoma patient subsets, so that valid conclusions may be drawn from the comparison of factors such as gender (male vs. female), age, number of microscopic vs. macroscopic nodes and location of primary tumor, among others.

Moreover, although our phase III trial demonstrated no difference in DFI or OS between patients receiving VMO and those receiving $\mathrm{V}$, our long-term results are in clear opposition to the vaccine trial conducted by Morton et al (37). These authors investigated Canvaxin, an antigen-rich, allogeneic whole-cell vaccine developed from three melanoma cell lines, which had appeared promising in phase I and II trials. However, in October 2005, CancerVax announced the discontinuation of its phase III clinical trial of Canvaxin in patients with stage III melanoma, since it was determined that Canvaxin-treated patients [those that received bacillus Calmette-Guérin (BCG) plus allogeneic melanoma cell vaccine], exhibited no improved survival benefit compared to patients that received BCG plus placebo. This was not the case in our trial, since the FDA did not detect any marked differences in OS or DFS among the $\mathrm{VMO}$ and $\mathrm{V}$ treatment groups. In fact, at the three-year mark, VMO showed a $10 \%$ survival advantage compared to $\mathrm{V}$, thus allowing for the continuation of the trial (31). However, at the five-year mark, the two arms were similar.

Another melanoma vaccine trial, which used a somewhat similar vaccine to ours, was one that Hersey and associates 
developed and further conducted as a prospective, randomized, multicenter trial, with the treatment arm consisting of vaccinia melanoma cell lysates (VMCL) and the control arm being a 'true' placebo (i.e., no-treatment arm) (38). The results of this trial showed that patients who were administered the vaccine did not exhibit a statistically significant improvement in OS or relapse-free survival, compared to the no-treatment arm. Although no statistically significant observations were made in the clinical trial conducted by Hersey et al, it is difficult to directly compare the results of our trial with theirs, since our vaccine consisted of four allogeneic melanoma cell lines with significant immunological activity $(24,25)$, as opposed to their single allogeneic melanoma cell line vaccine.

This phase III trial demonstrated an unexpected long-term survival in both arms; however, there are components of the vaccine that require improvement, which may allow for the optimal presentation of MAAs to the immune system, i.e., the inclusion of melanoma cell lines expressing HLA antigens. Over the past few years, we have designed a new vaccine incorporating the latest advances in cell and molecular biology. We have previously demonstrated the ability of dendritic cells (DCs) pulsed with an IL-2 gene-encoded vaccinia virus (VV) melanoma oncolysate (DC-IL-2VMO or DC-MelVac) to generate a cellular immune response in vitro (39) and in murine tumor models (40). This second generation melanoma vaccine possesses key immunogenic properties that may render it a potent therapeutic agent in the treatment of patients with stage III and IV melanoma and was approved by the FDA for a phase I trial in February 2005. We are currently in the development phase of this second generation melanoma vaccine, which consists of components that increase its adjuvanticity, compared to the first generation melanoma vaccine. Furthermore, we are striving for the complete characterization of the antigens within the VMO preparation, which is pivotal for developing an effective pan-antigen multivalent vaccine, using vaccinia virus as an adjuvant.

\section{Aknowledgements}

This clinical trial was funded by NCI (RO1CA4538-01A1).

\section{References}

1. Haass NK, Smalley KS and Herlyn M: The role of altered cell-cell communication in melanoma progression. J Mol Histol 35: 309-318, 2004.

2. Inamdar GS, Madhunapantula SV and Robertson GP: Targeting the MAPK pathway in melanoma: why some approaches succeed and other fail. Biochem Pharmacol 80: 624-637, 2010.

3. Siegel R, Ward E, Brawley O and Jemal A: Cancer statistics, 2011 the impact of eliminating socioeconomic and racial disparities on premature cancer deaths. CA Cancer J Clin 61: 212-236, 2011.

4. Atkins MB, Hsu J, Lee S, Cohen GI, Flaherty LE, Sosman JA, Sondak VK and Kirkwood JM; Eastern Cooperative Oncology Group: Phase III trial comparing concurrent biochemotherapy with cisplatin, vinblastine, dacarbazine, interleukin-2, and interferon alfa- $2 \mathrm{~b}$ with cisplatin, vinblastine, and dacarbazine alone in patients with metastatic malignant melanoma (E3695): a trial coordinated by the Eastern Cooperative Oncology Group. J Clin Oncol 26: 5748-5754, 2008

5. Bedikian AY, Millward M, Pehamberger H, Conry R, Gore M, Trefzer U, et al: Bcl-2 antisense (oblimersen sodium) plus dacarbazine in patients with advanced melanoma: the Oblimersen Melanoma Study Group. J Clin Oncol 24: 4738-4745, 2006.

6. Weber J: Immunotherapy for melanoma. Curr Opin Oncol 23: $163-169,2011$.
7. Block MS, Suman VJ, Nevala WK, Kottschade LA, Creagan ET, Kaur JS, et al: Pilot study of granulocyte-macrophage colonystimulating factor and interleukin-2 as immune adjuvants for a melanoma peptide vaccine. Melanoma Res 21: 438-445, 2011

8. Hodi FS, O'Day SJ, McDermott DF, Weber RW, Sosman JA, Haanen JB, et al: Improved survival with ipilimumab in patients with metastatic melanoma. N Engl J Med 363: 711-723, 2010.

9. Flaherty KT, Yasothan U and Kirkpatrick P: Vemurafenib. Nat Rev Drug Discov 10: 811-812, 2011.

10. McCardle TW, Messina JL and Sondak VK: Completely regressed cutaneous melanocytic lesion revisited. Semin Oncol 36: 498-503, 2009.

11. Overwijk WW and Restifo NP: Autoimmunity and the immunotherapy of cancer: targeting the 'self' to destroy the 'other'. Crit Rev Immunol 20: 433-450, 2000.

12. Pittet MJ, Zippelius A, Valmori D, Speiser DE, Cerottini JC and Romero P: Melan-A/MART-1-specific CD8 T cells: from thymus to tumor. Trends Immunol 23: 325-328, 2002.

13. Gnjatic S, Nishikawa H, Jungbluth AA, Güre AO, Ritter G, Jäger E, et al: NY-ESO-1: review of an immunogenic tumor antigen. Adv Cancer Res 95: 1-30, 2006.

14. Boon T: Tumor antigens recognized by cytolytic $\mathrm{T}$ lymphocytes: present perspectives for specific immunotherapy. Int J Cancer 54: 177-180, 1993.

15. Rosenberg SA: A new era of cancer immunotherapy: converting theory to performance. CA Cancer J Clin 49: 70-73, 1999.

16. Reynolds SR, Celis E, Sette A, Oratz R, Shapiro RL, Johnston D, Fotino $\mathrm{M}$ and Bystryn JC: Identification of HLA-A*03, A*11 and B*07-restricted melanoma-associated peptides that are immunogenic in vivo by vaccine-induced immune response (VIIR) analysis. J Immunol Methods 244: 59-67, 2000.

17. Reynolds SR, Celis E, Sette A, Oratz R, Shapiro RL, Johnston D, Fotino $\mathrm{M}$ and Bystryn JC: HLA-independent heterogeneity of CD8+ T cell responses to MAGE-3, Melan-A/MART-1, gp100, tyrosinase, MC1R, and TRP-2 in vaccine-treated melanoma patients. J Immunol 161: 6970-6976, 1998.

18. Reynolds SR, Oratz R, Shapiro RL, Hao P, Yun Z, Fotino M, Vukmanović S and Bystryn JC: Stimulation of CD8+ T cell responses to MAGE-3 and Melan A/MART-1 by immunization to a polyvalent melanoma vaccine. Int J Cancer 72: 972-976, 1997.

19. Baurain J, Stas M, Hammouch F, Gillain A, Feyens A, Van Baren N, et al: Association of primary melanoma ulceration and clinical benefit of adjuvant vaccination with tumor-specific antigenic peptides. J Clin Oncol 27: abs. 3022, 2009.

20. Kruit WH, van Ojik HH, Brichard VG, Escudier B, Dorval T, Dréno B, et al: Phase 1/2 study of subcutaneous and intradermal immunization with a recombinant MAGE-3 protein in patients with detectable metastatic melanoma. Int J Cancer 117: 596-604, 2005.

21. Slingluff CL Jr, Petroni GR, Olson WC, Smolkin ME, Ross MI, Haas NB, et al: Effect of granulocyte/macrophage colony-stimulating factor on circulating CD8+ and CD4+ T-cell responses to a multipeptide melanoma vaccine: outcome of a multicenter randomized trial. Clin Cancer Res 15: 7036-7044, 2009.

22. Filipazzi P, Pilla L, Patuzzo R, Castelli C, Maurichi A, Tragni G, et al: Adjuvant multipeptide vaccination in high-risk early melanoma patients. J Clin Oncol 26: abs. 3014, 2008.

23. Schwartzentruber DJ, Lawson DH, Richards JM, Conry RM, Miller DM, Treisman J, et al: gp100 peptide vaccine and interleukin-2 in patients with advanced melanoma. N Engl J Med 364: 2119-2127, 2011.

24. Wallack MK, Steplewski Z, Koprowski H, Rosato E, George J, Hulihan B and Johnson J: A new approach in specific, active immunotherapy. Cancer 39: 560-564, 1977.

25. Wallack MK: Specific active immunotherapy with vaccinia oncolysates. In: Tumor Progression. Crispen R (ed). Elsevier/ North Holland Inc., New York, pp.277-287, 1980.

26. Berthier-Vergnes O, Portoukalian J, Lefthériotis E and Doré JF: Induction of IgG antibodies directed to a M(r) 31,000 melanoma antigen in patients immunized with vaccinia virus melanoma oncolysates. Cancer Res 54: 2433-2439, 1994.

27. Shimizu Y, Hasumi K, Masubuchi K and Okudaira Y: Immunotherapy of tumor-bearing mice utilizing virus help. Cancer Immunol Immunother 27: 223-227, 1988.

28. Wallack MK, Meyer M, Bourgoin A, Doré JF, Leftheriotis E, Carcagne $J$ and Koprowski $\mathrm{H}$ : A preliminary trial of vaccinia oncolysates in the treatment of recurrent melanoma with serologic responses to the treatment. J Biol Response Mod 2: 586-596, 1983. 
29. Wallack MK, McNally KR, Leftheriotis E, Seigler H, Balch C, Wanebo H, Bartolucci AA and Bash JA: A Southeastern Cancer Study Group phase I/Il trial with vaccinia melanoma oncolysates. Cancer 57: 649-655, 1986.

30. Wallack MK, Bash JA, Leftheriotis E, Seigler H, Bland K, Wanebo $\mathrm{H}$, Balch $\mathrm{C}$ and Bartolucci AA: Positive relationship of clinical and serological responses to vaccinia melanoma oncolysate. Arch Surg 122: 1460-1463, 1987.

31. Wallack MK, Sivanandham M, Balch CM, Urist MM, Bland KI, Murray D, et al: A phase III randomized, double-blind multiinstitutional trial of vaccinia melanoma oncolysate-active specific immunotherapy for patients with stage II melanoma. Cancer 75: 34-42, 1995.

32. Wallack MK, Sivanandham M, Ditaranto K, Shaw P, Balch CM, Urist MM, et al: Increased survival of patients treated with a vaccinia melanoma oncolysate vaccine. Second interim analysis of data from a phase III, multi-institutional trial. Ann Surg 226: 198-206, 1997.

33. Wallack MK, Sivanandham M, Balch CM, Urist MM, Bland KI, Murray D, et al: Surgical adjuvant active specific immunotherapy for patients with stage III melanoma: the final analysis of data from a phase III, randomized, double-blind, multicenter vaccinia melanoma oncolysate trial. J Am Coll Surg 187: 69-79, 1998.

34. Roenigk HH Jr, Deodhar S, Jacques R and Burdick K: Immunotherapy of malignant melanoma with vaccinia virus. Arch Dermatol 109: 668-673, 1974.
35. Linge C, Gewert D, Rossmann C, Bishop JA and Crowe JS: Interferon system defects in human malignant melanoma. Cancer Res 55: 4099-4104, 1995.

36. Joosse A, de Vries E, Eckel R, Nijsten T, Eggermont AM, Hölzel D, Coebergh JW and Engel J; Munich Melanoma Group: Gender differences in melanoma survival: female patients have a decreased risk of metastasis. J Invest Dermatol 131: 719-726, 2011.

37. Morton DL, Hsueh EC, Essner R, Foshag LJ, O'Day SJ, Bilchik A, et al: Prolonged survival of patients receiving active immunotherapy with Canvaxin therapeutic polyvalent vaccine after complete resection of melanoma metastatic to regional lymph nodes. Ann Surg 236: 438-449, 2002.

38. Hersey P, Coates AS, McCarthy WH, Thompson JF, Sillar RW, McLeod R, et al: Adjuvant immunotherapy of patients with high-risk melanoma using vaccinia viral lysates of melanoma: results of a randomized trial. J Clin Oncol 20: 4181-4190, 2002.

39. Aydin N, Jack A, Montenegro G, Boyes C, Alam K and Wallack MW: Expression of melanoma-associated antigens in human dendritic cells pulsed with an interleukin-2 gene encoded vaccinia melanoma oncolysate (rIL-2VMO). Cancer Biol Ther 5: 1654-1657, 2006.

40. Jack AM, Aydin N, Montenegro G, Alam K and Wallack MK: A novel dendritic cell-based cancer vaccine produces promising results in a syngenic CC-36 murine colon adenocarcinoma model. J Surg Res 139: 164-169, 2007. 